\title{
Comprehensive Care Models for Cardiometabolic Disease
}

\author{
Cara Reiter-Brennan $^{1,2} \cdot$ Omar Dzaye $^{1,2} \cdot$ Dorothy Davis $^{1} \cdot$ Mike Blaha $^{1,3} \cdot$ Robert H. Eckel $^{4}$
}

Accepted: 8 January 2021 / Published online: 24 February 2021

(C) The Author(s), under exclusive licence to Springer Science+Business Media, LLC part of Springer Nature 2021

\begin{abstract}
Purpose of Review The high burden of cardiovascular disease and the simultaneous obesity pandemic is placing an extraordinary strain on the health care system. In the current siloed care model, patients with cardiometabolic disease receive only fractionated care from multiple specialists, leading to insufficient treatment, higher costs, and worse outcomes.

Recent Findings The imminent need for a new care model calls for the creation of a distinct cardiometabolic specialty in conjunction with a cardiometabolic outpatient clinic dedicated to the comprehensive cardiometabolic care. The cardiometabolic clinic would consist of a diverse range of professionals relevant to comprehensive treatment.

Summary The outpatient clinic we envision here would facilitate an interdisciplinary collaboration between specialists and deliver prevention-focused treatment to patients at risk/established cardiometabolic disease.
\end{abstract}

Keywords Cardiology $\cdot$ Diabetes $\cdot$ Endocrinology $\cdot$ Metabolism $\cdot$ Prevention

\begin{tabular}{|c|c|}
\hline \multicolumn{2}{|c|}{ Abbreviations } \\
\hline ASCVD & A therosclerotic cardiovascular disease \\
\hline AWV & Annual wellness visit \\
\hline BMI & Body mass indey \\
\hline CDCES & $\begin{array}{l}\text { Certified diabetes care and education } \\
\text { specialist }\end{array}$ \\
\hline $\mathrm{CHD}$ & Coronary heart disease \\
\hline CKD & Chronic kidney disease \\
\hline CVD & Cardiovascular disease \\
\hline CVOTS & Cardiovascular outcome trials \\
\hline DASH & Dietary approach to stop hypertension \\
\hline DM & Diabetes mellitus \\
\hline DSMES & $\begin{array}{l}\text { Diabetes self-management education } \\
\text { and support }\end{array}$ \\
\hline \multicolumn{2}{|c|}{$\begin{array}{l}\text { This article is part of the Topical Collection on Diabetes and } \\
\text { Cardiovascular Disease }\end{array}$} \\
\hline \multicolumn{2}{|c|}{$\begin{array}{l}\triangle \text { Robert H. Eckel } \\
\text { robert.eckel@cuanschutz.edu }\end{array}$} \\
\hline \multicolumn{2}{|c|}{$\begin{array}{l}\text { Cara Reiter-Brennan } \\
\text { cara.reiter-brennan@charite.de }\end{array}$} \\
\hline \multicolumn{2}{|c|}{$\begin{array}{l}\text { Omar Dzaye } \\
\text { odzaye@jhmi.edu }\end{array}$} \\
\hline \multicolumn{2}{|c|}{ Dorothy Davis } \\
\hline $\begin{array}{l}\text { Mike I } \\
\text { mblah }\end{array}$ & \\
\hline
\end{tabular}

$\begin{array}{ll}\text { FDA } & \text { Food and Drug Administration } \\ \text { GLP-1 RA } & \text { Glucagon-like peptide-1 receptor agonist } \\ \text { HF } & \text { Heart failure } \\ \text { HbA1c } & \text { Hemoglobin A1c } \\ \text { ICU } & \text { Intensive care unit } \\ \text { LDL-C } & \text { Low-density lipoprotein cholesterol } \\ \text { MI } & \text { Myocardial infarct } \\ \text { MNT } & \text { Medical nutrition therapy } \\ \text { NAFLD } & \text { Non-alcoholic fatty liver disease } \\ \text { SGLT-2 } & \text { Sodium-glucose transporter-2 } \\ \text { T1DM/T2DM } & \text { Type 1/type 2 diabetes mellitus }\end{array}$

1 Johns Hopkins Ciccarone Center for the Prevention of Cardiovascular Disease, Johns Hopkins University School of Medicine, Baltimore, MD, USA

2 Department of Radiology and Neuroradiology, Charité, Berlin, Germany

3 Welch Center for Prevention, Epidemiology and Clinical Research, Johns Hopkins University, Baltimore, MD, USA

4 Division of Endocrinology, Metabolism and Diabetes and Division of Cardiology, University of Colorado School of Medicine, 18582 Stone Gate Dr., Morrison, CO 80465, USA 


\section{Introduction}

The prevalence of patients with chronic metabolic and with cardiovascular multi-comorbidities has been increasing steadily over the course of the last decades. Cardiovascular disease (CVD) is the main cause of morbidity and mortality among patients with type 2 diabetes mellitus (T2DM) [1]. Affecting 85.6 million Americans, 219 billion dollars are spent on CVD each year [2]. Often, both diseases coexist together, with as many as two-thirds of patients with CVD being dysglycemic [3]. However, metabolic disorders of patients with CVD frequently remain undiagnosed and are untreated, resulting in a higher incidence of cardiovascular death [4]. Cardiometabolic disease already places substantial strain on the health care system. Individuals with diabetes have 2.3 higher medical costs when adjusted for age and gender [1]. In light of increasing obesity prevalence, the aging population, and rising medical expenditures, this will only expand in the future.

However, in our present health care system, the optimal management of these complex cardiometabolic patients is compromised. In our traditional siloed care model, there is no "main" specialist coordinating treatment, as cardiologists and endocrinologists often work with little overlap. Other important aspects of cardiometabolic therapy such as lifestyle intervention and psychological counseling are not adequately addressed. As a result, cardiometabolic patients receive fragmented care with redundant diagnostic testing and higher costs and are at risk of drug-drug interaction and, most importantly, adverse CVD events.

Fortunately, we have witnessed advancements on the front of cardiometabolic pharmacology. Novel, evidence-based "cardiometabolic drugs" initially approved for the treatment of T2DM have proven to treat not only diabetes but also CVD. These cardiometabolic drugs further blur the lines between traditional specialties, emphasizing the need to rethink current siloed care models.

As a solution to this urgent issue, we propose the development of a distinct cardiometabolic specialty addressing the needs of the cardiometabolic patient. The education of cardiometabolic physicians would start early, with core concepts of cardiometabolic disease introduced in medical school. After a general internal residency program, physicians could specialize in cardiometabolic medicine by completing a $2-3$-year cardiometabolic specialist training program. Finally, the cardiometabolic physician would have the opportunity to work in an outpatient clinic dedicated to the cardiometabolic patient (Fig. 1). In this publication, we formulate a prototype for a cardiometabolic clinic distinctly addressing the needs of the cardiometabolic patient. This clinic would consist of a well-coordinated team of specialists of a cardiometabolic physician, cardiometabolic nurses, dieticians, rehabilitation physicians, certified diabetes care and education specialist (CDCES), and psychologists. Through a collaborative, interdisciplinary approach to this care model, we would hope to optimize treatment of the cardiometabolic patient, improve outcomes, and reduce medical expenditure.

\section{Recent Trends in Epidemiology of Cardiometabolic Disease}

Recent trends in CVD-related mortality are concerning. While the USA has witnessed a considerable decrease of death from CVD in the past decades, this trend seems to be dissipating. However, age-adjusted CVD mortality has remained at $0.5 \% / y e a r$, while other areas have witnessed a continuous decrease in mortality (e.g., cancer decreased $1.5 \%$ annual between 2000 and 2015) [5, 6]. Indeed, CVD accounts for 17.3 million deaths a year globally, but according to a 2014 study, is expected to cause over 23.6 million deaths per year by 2030 [7]. Reduced improvement of CVD mortality is most likely to be attributed to the alarming increase in obesity prevalence. In the USA, $39.4 \%$ of adults $\geq 20$ years of age are obese [8]. By 2030, estimates suggest that one in two Americans will suffer from obesity [9]. Obese individuals, particularly those with metabolic syndrome, are at higher risk of diabetes and adverse CVD outcomes [10]. Obesity is a main driver of diabetes. Among US adults, $10.5 \%$ are diagnosed with diabetes while another $37.6 \%$ are pre-diabetic [11]. Data from National Health and Nutrition Examination Survey (NHANES) suggest that in the last three decades, blood glucose health has deteriorated in obese individuals, leading to a worsening of CVD [12]. Individuals with diabetes are associated with a two times higher risk of coronary heart disease (CHD) and 2.3 times higher for ischemic stroke [13]. Estimates suggest that $10 \%$ of vascular deaths are attributed to diabetes and two-thirds of patients with T2DM die from CVD [13, 14]. Indeed, diabetes is often unrecognized in patients with CVD. In a study of left ventricular dysfunction, $11 \%$ of patients with HF had undiagnosed T2DM [15]. Finally, apart from cardiovascular comorbidities, CKD is one of the most relevant co-diagnosis of diabetes. In the developed world, diabetes is the primary cause of CKD and patients with diabetes and CKD have a markedly elevated risk of CVD [16, 17]. Albuminuria is associated with an atherogenic lipoprotein profile of elevated TGs, low HDL-C, and a shift in LDL-C, with a higher proportion of small, high-density lipoproteins $[18,19]$. However, current guidelines are inconstant in assessment of cardiovascular risk of patients with CKD, prohibiting adequate preventive CVD treatment. 


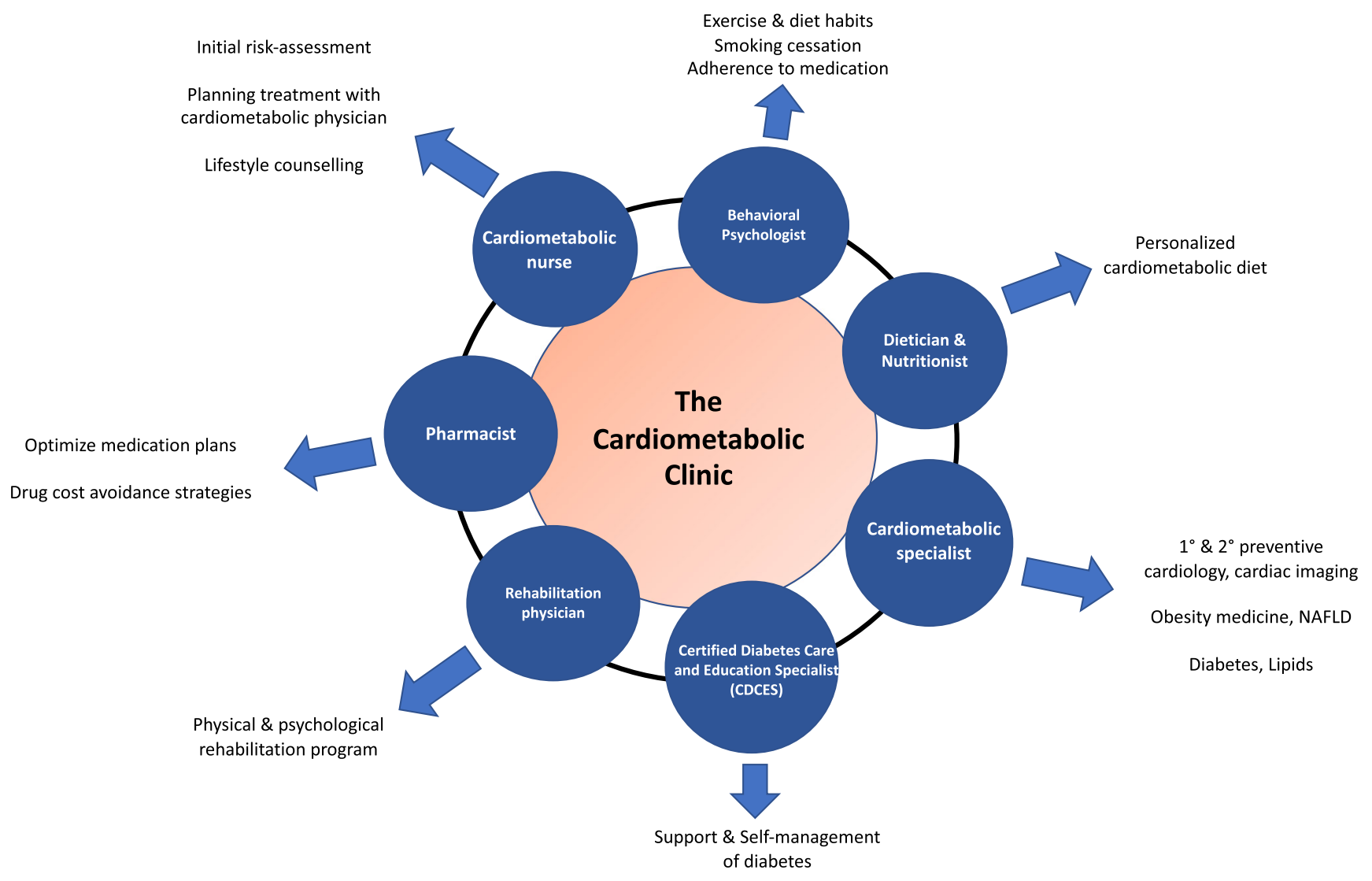

Fig. 1 The cardiometabolic clinic

\section{Advances in Cardiometabolic Drugs and Their Limited Use in Clinical Care}

Fortunately, we have witnessed a paradigm shift in the development of pharmaceutical drugs targeting T2DM as well as CVD. Cardiovascular outcome trials (CVOTs) demonstrated the cardiovascular benefits of SGLT-2 inhibitors and GLP1RAs for patients with T2DM (and in some cases without T2DM). The Food and Drug Administration (FDA) has now approved the use of antidiabetic agents to lower risk of a composite of major adverse cardiac events (liraglutide [20], canagliflozin [21••], semaglutide [22], dulaglutide [23•]), cardiovascular death (empagliflozin [24]), and HF (dapagliflozin [25]). This recommendation is echoed in recent professional guidelines. SGLT-2 inhibitors were shown to be particularly beneficial for patients with HF and chronic kidney disease (CKD) and should be subscribed to patients with T2DM and CKD or HF $[21,26]$. If obesity is a central concern, GLP-1 RAs are recommended, as they are currently the most potent anti-diabetic drug for weight loss [27].

However, despite guideline recommendation and FDA approval, these evidence-based therapies are underused. A cohort study investigating contemporary use of guideline recommended medication among patients with atherosclerotic cardiovascular disease (ASCVD) and diabetes found that only 9.0\% of individuals received SGLT-2 inhibitors and 7.9\% were subscribed GLP-1 RAs [28]. Indeed, investigators observed optimal pharmaceutical therapy in only $6.9 \%$ of cases [28]. These agents are a vital opportunity to improve care and reduce cardiovascular events in these patients. We believe that training physicians to appropriately treat cardiometabolic patients would lower barriers of subscription of these agents.

\section{Current Siloed Care Models}

While the pharmaceutical sector has developed "cardiometabolic" agents, physician education and clinical care is still divided into isolated cardiology and endocrinology programs, with little overlap between disciples. Some argue that primary care physicians should be adept in managing care for patients with ASCVD and diabetes. In reality, primary care providers battle time constraints and face competing demands during routine primary care visits with little bandwidth to comprehensively treat the cardiometabolic patient [29॰]. We are also facing a severe shortage of primary care physicians. Only onequarter of medical students plan on pursing primary care [30]. While it may seem intuitive for cardiologists to lead the 
management of cardiometabolic patients, traditional cardiology training does not prepare physicians for glycemic management, prescribing weight loss drugs or adequate lifestyle counseling. Cardiologists may feel uncomfortable monitoring side effects of novel cardiometabolic drugs like SGLT-2 inhibitors and GLP1-RAs, in addition to the complex management of ASCVD drugs like PCSK9 inhibitors, beta blockers, renin-angiotensin-aldosterone system inhibitors, or anticoagulant regimes. As a result of this fractionated care, fewer than $20 \%$ of patients with diabetes reach all three guideline recommended targets for HbA1c, blood pressure, and low-density lipoprotein-cholesterol [31]. Ultimately, this issue calls for professional experts and related health care team in cardiometabolic disease to provide comprehensive, guidelinerecommended treatment of these complex patients.

\section{Education in Cardiometabolic Medicine}

\section{Medical School}

The foundations of cardiometabolic medicine should be laid in medical school, and students who are interested can choose further training as a cardiometabolic specialist during residency. Current medical education teaches disease as single entities, rather than emphasizing that chronic conditions often occur simultaneously and multimorbid patients are the norm. Teaching ASCVD and T1DM/T2DM in one holistic cardiometabolic course instead of in separate system blocks would allow students to better grasp the interdisciplinary nature of disease entities and prepare students for multimorbid patients. Education in cardiometabolic medicine in medical school would incorporate lessons on lifestyle counseling and behavioral medicine: an area underdeveloped in current curricula.

\section{The Cardiometabolic Specialist}

Following medical school and 2-3 years of general internal medicine house-staff training, we envision a cardiometabolic training program consisting of a composite of cardiology, endocrinology, and other specialties relevant to the cardiometabolic patient.

Primary and secondary ASCVD prevention would be the focus of the cardiology component of this program. Training in electrophysiology, interventional cardiology, advanced HF, or cardiac transplantation would however not be part of this curriculum. Rotations in hypertension clinics and vascular medicine would allow for training for treatment of severe hypertension. Physicians would be able to correctly diagnose resistant hypertension by excluding pseudoresistance and screening for secondary hypertension, as well as assessing organ damage. Compared to traditional endocrinology specialist training, more emphasis would be placed on lessons on the complex pharmacological treatment of resistant hypertension and life-style intervention. In addition, some experience in hepatology would further broaden the experience of cardiometabolic medicine that relates non-alcohol fatty liver disease (NAFLD) and non-alcoholic steatohepatitis (NASH) to ASCVD [32]. The endocrinology aspects of this specialization program would focus on obesity, metabolic syndrome, T1DM and T2DM, lipid and lipoprotein disorders, endocrine causes of hypertension, and lifestyle. Physicians would be trained in advanced glucose management by covering the administration of basal/bolus insulin, insulin infusion pumps, and glucose sensors. Other traditional topics regarding diseases of thyroid, hypothalamic-pituitary-adrenal axis, reproductive endocrinology, or metabolic bone disease, including parathyroid disorders would not be covered during the cardiometabolic training program.

In addition to endocrinology and cardiology training, the curriculum would allow enough time for training in lifestyle counseling. Physicians would be adept in smoking cessation therapy, exercise physiology, and nutrition counseling. Behavioral intervention training would provide trainees with skills to enhance therapy adherence, physician-patient communication, and motivational interviewing. A substantial component of training would be spent on the most relevant topics of obesity medicine, focusing particularly on NAFLD and NASH. Rotations through obesity clinics would provide physicians with training in obesity pharmacology, as well as when and how to refer patients to bariatric surgery and postoperative management.

\section{The Cardiometabolic Clinic}

\section{The Cardiometabolic Team}

Finally, we envision the establishment of a cardiometabolic outpatient clinic with a multidisciplinary team consisting of a cardiometabolic physician, behavioral psychologists, nutritionists, certified diabetes care and education specialists (CDCES), and specialized rehabilitation physicians. These professionals as well as three cardiometabolic nurses would form a cohesive, interdisciplinary team. The cardiometabolic clinic would be managed by two administrative personnel. In a well-groomed high-volume clinic, this is the minimum number of staff required but can be expanded as needed. As the clinic would provide clinical training, students and residents would be a welcomed part of the team.

\section{Practicalities}

The cardiometabolic nurse would triage new patients and provide an initial assessment to decide which type of professional the patient requires. Triaging would incorporate 
cardiovascular risk assessment when indicated through cardiac imaging, as well as initial bloodwork. After consultation with the cardiometabolic physician, a personalized treatment plan for the patient would be created. Through this type of holistic assessment of patients, high-risk individuals can be quickly identified. We envision the cardiometabolic clinic as a continuous care model, where patients return for regular check-ups and counseling sessions after initial referral.

The success of treating complex cardiometabolic patients relies on the successful collaboration between team members of the cardiometabolic clinic. Other medical specialties report a multidisciplinary approach as the most efficient strategy to handling complex patients [33]. This approach would be fostered by daily team meetings discussing therapeutic plans and new research evidence regarding cardiometabolic disease. Results from a meta-analysis suggest that poor coordination of and information transfer within a medical team reduced patient satisfaction in outpatient clinical settings [34]. In a survey of chronically ill US adults, $30 \%$ reported missing medical records or duplication of tests during outpatient treatment [35]. A well-coordinated cardiometabolic outpatient clinic would reduce such errors, improving outcome and patient satisfaction.

\section{Subspecialties Working at the Clinic}

\section{Cardiometabolic Specialist}

The cardiometabolic physician's work would primarily consist of a composite of endocrinology and cardiology, with a focus on primary and secondary prevention of CVD.

At the beginning of treatment, cardiovascular imaging (echocardiography, stress testing, and coronary computed tomography) to assess ASCVD would be individualized and at times used to make nuanced decisions about cardiovascular risk. The cardiometabolic clinic would also offer transthoracic echocardiography to quickly and easily assess patients with suspect structural heart disease. Transesophageal echocardiography, magnetic resonance imaging, and nuclear imaging are beyond the scope of the cardiometabolic physician. Additionally, the cardiometabolic physician would manage lipids and have experience in treating complex patients with severe hypertriglyceridemia, statin intolerance or genetic lipid and lipoprotein disorders such as familial hypercholesterolemia. Patients would also be treated for hypertension including resistant. The ramification of causes of resistant hypertension demands a systematic and multidisciplinary approach in diagnosis and treatment [36]. This would be facilitated by the well-coordinated integrative team of the cardiometabolic clinic. A routine part of the cardiometabolic physician's work would be assessment of CKD and the associated cardiovascular risk through albuminuria measurement. The cardiometabolic physician possessing endocrinology and cardiology expertise could competently manage CKD as well as any cardiovascular comorbidities according to most recent evidence. Physicians would be adept in glycemic management of patients, from coordinating prescriptions of metformin, GLP-1 RAs, SGLT-2 inhibitors, thiazolidinediones, and other drug classes for the treatment of diabetes to complex insulin administration schemes and new diabetes technologies. The cardiometabolic specialist would also be in charge of obesity management by prescribing and individualized approach to lifestyle management, weight loss medications, and when and how to refer patients to metabolic surgeons and be knowledgeable in managing post-bariatric surgery patients.

\section{The Cardiometabolic Nurse}

We envision the cardiometabolic nurse to work side by side with the cardiometabolic physician. Evidence suggests that diabetes and CVD care quality was comparable between physicians and advanced care providers (nurse practitioners or physician assistants) delivered in a primary care setting [37]. The cardiometabolic nurse would conduct an initial comprehensive health assessment, including cardiac and metabolic history, screening for risk factors, taking blood tests, and referring patients to cardiac imaging tests. After discussion with the cardiometabolic specialist, the cardiometabolic nurse would contact patients to discuss lab and imaging results and help direct patients to follow-up appointments and when necessary to other subspecialists. With respect to the continuity of care model, nurses would contact patients regularly to inquire about treatment progress and remind patients of appointments. As the cardiometabolic clinic would grow, clerks would take over scheduling routine appointments while cardiometabolic nurses would ensure continuity of care by discussing lifestyle goals and progress with patients and decide if follow-up appointments or referrals are necessary. Preventive care in the form of intensive lifestyle intervention should form an essential part of the new model of care underpinning the cardiometabolic clinic. In lifestyle counseling sessions, nurses will discuss exercise and diet as well as smoking cessation plans with patients. For some patients, these lifestyle counseling sessions will suffice. If more intensive lifestyle interventions are needed, the cardiometabolic nurse can refer patients to the behavioral psychologist, nutritionist, or exercise physiologist. An excellent lifestyle counseling program is vital for the successful treatment of cardiometabolic disease and will distinguish this clinic from other programs. An estimated $40 \%$ of deaths in USA are attributed to poor lifestyle choices, but physicians only provide lifestyle counseling in $34 \%$ of cases $[38,39]$. The importance of physicians to effective lifestyle assessment of patients with cardiovascular disease has been previously emphasized [40]. 


\section{Behavioral Psychologist}

Some patients may require regular sessions with a behavioral psychologist. CVD outcomes are highly linked to psychological factors. For example, depression is a known risk factor for CHD and HF and a relevant comorbidity of diabetes [41, 42]. Behavioral change therapy would also address nonadherence to prescribed medication. Only half of patients without CHD and one-third with CHD adhere to their drugs prescribed [43]. Using techniques such as cognitive behavioral therapy techniques and motivational interviewing, behavioral change psychologists would facilitate lasting transformation in patient's exercise and eating habits and expedite smoking cessation. Evidence suggests that structured behavioral support by a psychological specialist is more effective for smoking cessation therapy than counseling by nurse practitioners or pharmacist [44]. Smoking cessation medication acts synergistically with behavioral consultation and should be subscribed to patients [45].

\section{Nutritionists}

Nutritionists are an important part of lifestyle management and need to be available to counsel patients regarding dietary interventions to optimize cardiometabolic health. There is a growing consensus that a cardioprotective diet consists of a diverse inclusion of healthful, nutrient-dense foods and is one of the most effective methods to prevent and treat CVD, obesity, and diabetes [46]. Dietary patterns such as the DASH (dietary approach to stop hypertension) diet and Mediterranean style diets can reduce ASCVD risk, improve blood lipids and blood pressure, and reduce inflammation [47, 48]. Evidence suggests that response to diet needs to be personalized, rather than focusing on a specific macro- and micro-nutrient distribution for each patient with diabetes. Thus, dietary counseling should take comorbidities, current eating patterns, preferences, and sociocultural factors into consideration. Patients with diabetes in particular profit from sessions with nutritionists, as choosing the right foods is often the most challenging part of treatment. Diabetes-specific medical nutrition therapy (MNT) is highly effective and awarded A-level recommendation by the ADA Standards of Medical Care 2020 [49]. Conveyed by a registered dietician, MNT is associated with a $\mathrm{HbA} 1 \mathrm{C}$ decrease up from 0.3 to $2 \%$ in individuals with T2DM and $1.0-1.9 \%$ in patients with T1DM [50].

\section{Certified Diabetes Care and Education Specialist}

Certified Diabetes Care and Education Specialist (CDCES) will form an integral part of the cardiometabolic team. The primary goal of diabetes education is to provide knowledge and skills to facilitate self-care behavior and help patients make informed self-management decisions. This type of lifestyle management known as diabetes self-management education and support (DSMES) is associated with lower $\mathrm{HbAlc}$ [51], lower all-cause mortality risk [52], and improved quality of life [53]. Despite ample evidence of the benefits of DSMES and B-level guideline recommendation, this service is underutilized. Only $6.8 \%$ of privately insured and $5 \%$ Medicare beneficiaries with diabetes take part in diabetes education, even though covered by most insurances [54, 55]. Through integration of CDCES in cardiometabolic clinic, we hope to make diabetes self-management and education a consistent part of standardized diabetes care.

\section{Specialized Rehabilitation Physicians}

Patients with previous cardiac events would be able to visit a cardiac rehabilitation specialist. Especially since hospitalization for serious cardiovascular events has shortened, outpatient rehabilitation is becoming more important. However, of eligible patients with previous MI, only $14-35 \%$ participated in cardiac rehabilitation programs but use is higher when patients are referred to rehabilitation facilities [56]. The integration of rehabilitation facilities into the cardiometabolic clinic and adding it to the standardized cardiometabolic treatment program would increase use of rehabilitation and improve outcomes.

\section{Pharmacist}

Incorporating clinical pharmacists in primary care settings significantly increased the percentage of patients with controlled hypertension and reduced medical expenditures [57]. Interdisciplinary, team-based interventions, such as facilitated in the cardiometabolic clinic, are particularly effective in improving outcome. Results suggest that involving pharmacists or nurses in team-based care is the most potent strategy to successfully control blood pressure [58]. Apart from managing complex medication plans, pharmacists would assist with time-consuming tasks, like drafting appeal letters for nonformulary medications such as the PSCK9 inhibitor drugs. Finally, pharmacists can improve medication adherence by reducing drug expenditure, one of the major causes for nonadherence [59]. Pharmacists employed in cardiac out-patient clinics facilitated cost-saving interventions like discount programs and copay interventions, resulting in a yearly $852 \$$ cost avoidance per patient [60].

\section{Patient Referral}

Primary care physicians, endocrinologists, and cardiologists who are overwhelmed or with too little expertise in certain areas of cardiometabolic medicine would refer 
patients. Additionally, the cardiometabolic clinic would manage follow-up care after cardiometabolic surgery (bariatric, vascular, or cardiothoracic) as part of patient's rehabilitation program. Patient referral could also be initiated by nurse practitioners, who could educate patients on benefits of the cardiometabolic clinic.

\section{Example of Patients Benefitting from the Cardiometabolic Clinic}

\section{Type 1 Diabetes}

The cardiometabolic clinic would be particularly beneficial for patients with T1DM. Limited data, primarily in the form of observational studies, exist on assessment and sustenance of cardiovascular health in T1DM patients. Data from randomized controlled trials and cohort studies chiefly refer to T2DM patients or make no distinction between types [61]. As a result, the underlying pathogenesis of the relationship between CVD and T1DM is not well understood and CVD treatment of T1DM patients has mainly been adapted from observational data sets or CVOTs in T2DM. Through the implementation of a dedicated cardiometabolic clinic, physicians working there would see more T1DM patients with CVD or at high risk and thereby gain more expertise in effectively treating this disease constellation.

\section{Complex Patients}

Multi-morbid, complex patients with multiple risk factors especially require an interdisciplinary treatment approach and would benefit from the cardiometabolic clinic. For example, a traditional cardiologist would perhaps be unsure of optimal therapeutic strategy for a 56-year-old obese (BMI $34 \mathrm{~kg} / \mathrm{m}^{2}$ ) woman with T2DM patient taking four medications to treat diabetes (excluding insulin). Her levels of glycemia and lipids are equally worrisome, with $\mathrm{HbA} 1 \mathrm{c}$ of $8.5 \%$ and triglycerides of $350 \mathrm{mg} / \mathrm{dL}$, respectively. Her blood pressure is $150 / 88$ and the echocardiogram reveals HF with preserved ejection fraction. This patient suffers from multiple comorbidities which require coordinated care by a cardiologist, endocrinologist, and obesity specialist. In the cardiometabolic clinic, even patients like this complex patient would receive comprehensive care. The clinic would also provide high-quality care for high-risk, under-represented populations demanding more intensive consideration such as patients with immunodeficiency syndromes, cancer survivors, and mentally handicapped individuals [62].

\section{Boundaries}

The cardiometabolic clinic will focus on primary and secondary prevention of ASCVD and diabetes and will not offer some advanced treatments offered in typical cardiology and endocrinology clinics. Electrophysiology is not part of physician training, and therefore treatment of cardiac arrythmias will not take place in this clinic. Similarly, interventional cardiology as well as expertise regarding advanced HF or cardiac transplantation will not be offered. We envision an outpatient clinic, and therefore this setting would not be suitable for intensive care patients. For example, patients with advanced $\mathrm{HF}$ and possible complications such as pulmonary edema with acute respiratory failure or pneumonia would require hospitalization with likely admission to the intensive care unit (ICU). Similarly, patients with cardiogenic shock requiring intensive hemodynamic monitoring and ventilatory support in the form of intubation and mechanical ventilation would not be treated in the cardiometabolic clinic. Physicians will not treat endocrinological disease not connected to cardiometabolic diseases, such as thyroid disease, hypothalamic/pituitary disease, or metabolic bone disease. Diagnosis and treatment of disorders of the reproductive axis would also not be implemented in this setting. Despite the increasing prevalence of T2DM among children and the micro- and macro-vascular complications associated with early onset, pediatric endocrinologists would for now be better suited to treat this subgroup [63]. However, cardiometabolic physicians would be available for consultations for children with cardiometabolic disease.

\section{Cardiometabolic Clinic Within the Health System}

\section{Value-Based-Care}

The value-based care model reimburses physicians and health care organization by positive health outcomes. Health care systems in the USA and in much of the rest of the world are dominated by the "fee-for-service" model, which compensates physicians by number of tests and procedures, regardless of patient outcome. A value-based care model incentivizes clinics to deliver the most high-quality care to avoid any cost-intensive complications of cardiometabolic disease in the future. The cardiometabolic clinic is well suited for a value-based care model, as the preventive-care approach reduces progression of chronic cardiovascular and metabolic diseases. As a result, patients require fewer diagnostic tests and procedures, which reduces cost. A nationwide study of 120 payers commissioned by Change Healthcare found an average reduction of $5.6 \%$ of medical cost savings after adopting value-based care strategies [64]. Improved care quality was reported by $77 \%$ of payers. 


\section{Reducing Costs}

Comprehensive preventive care as the cardiometabolic clinic would provide lowers healthcare expenditure, primarily by reducing costly hospitalization. Costs for preventable hospitalizations by primary preventive care is estimated to exceed $30 \$$ billion annually [65]. Free ambulatory clinics often treat chronic illness of uninsured patients by regular monitoring, providing prescription medication and lab diagnostics [66]. A study using North Carolina hospital discharge data demonstrated that uninsured adults had a $8.0 \%$ reduced odds of hospitalization for conditions which could have been mitigated by appropriate primary care after receiving regular treatment from free ambulatory clinics [66]. By preventing hospitalization of conditions caused by cardiometabolic disease, the cardiometabolic clinic could be equally successful in reducing hospitalization and healthcare costs. Existing preventive care models with similar concepts to the cardiometabolic clinic demonstrated lower healthcare costs and improved clinical care quality for beneficiaries. For example, the annual wellness visit (AWV) eligible to Medicare recipients prioritizes preventive care elements, such as treating mental health conditions and screening for risk factors, rather than addressing acute complaints [67]. AWV was associated with a significant reduction of patient's healthcare costs, mainly driven by reduction of hospital acute care and hospital outpatient nonemergency department spending [67]. Additionally, the new comprehensive and well-coordinated model of care outlined here would reduce repeat diagnostic testing and trips to other clinics for patients, thereby reducing health care costs. An outpatient clinic would also be more profitable by reduced operating and staffing costs.

\section{Health Insurance}

Regular health insurance would cover treatments in the cardiometabolic clinic. However, all patients referred to the clinic would be referred as "new patients," as cardiometabolic medicine would be categorized as a separate specialty and not part of cardiology or endocrinology clinics.

\section{Example}

Our vision of the cardiometabolic clinic and its benefits is best delineated by an example. Take Mr. Morris, a 60-year old overweight (BMI 28) male with T2DM managed with basal insulin who suffered a myocardial infarct (MI) 2 years ago, who comes for his 15-min visit at his general practitioner's office. Of recent he has experienced shortness of breath on exertion and palpitations.

His blood pressure is elevated at 140/90 and his LDL-C level is $110 \mathrm{mg} / \mathrm{dL}$. His primary care physician would alter blood pressure and lipid-lowering medication as well as evaluate insulin therapy and antiplatelet medications. He might be able to carve out a couple of minutes to discuss lifestyle interventions including dietary modifications, but in light of other pressing matters, this would not be a priority. In addition, Mr. Morris would be referred to other specialists. The cardiologist may further alter blood pressure medications, statins and perhaps prescribe ezetimibe or a PCSK-9 inhibitor, despite previous changes already made by the primary care physician. The endocrinologist would be more proficient in insulin adjustments but may have limited experience in obesity pharmacotherapy and cardiovascular comorbidities. It is unclear which physician would prescribe "cardiometabolic" drugs like GLP-1 RAs or SGLT-2 inhibitors. At all three appointments, physicians would re-take his blood levels and blood pressure. However, most likely none of the specialists would be able to coordinate the complex management of diabetes, lipids, blood-pressure, obesity and existing CVD and related lifestyle and medications interact in cardiometabolic patients like Mr. Morris.

This example outlines the unfortunate reality of the fractionated care many patients with cardiometabolic disease receive. In contrast, in the cardiometabolic clinic, Mr. Morris would receive a comprehensive and personalized cardiometabolic treatment program. Since the cardiometabolic physician is in charge of diagnostics and prescribing lifestyle modifications and medications, there is evidence-based evaluation and reduced chance of unnecessary testing or procedures. In addition, because of intensive counseling sessions with the cardiometabolic nurse as well as nutritionists, rehabilitation physicians, and behavioral psychologists, Mr. Morris would receive holistic advice on diet and physical activity as well as addressing psychological factors of cardiometabolic disease. Moreover, Mr. Morris will feel much more involved in his treatment, and working with the cardiometabolic team can make informed decisions to follow.

\section{Conclusion}

The imminent need to create a new care model to treat patients with cardiometabolic disease is one of the most important tasks in our current health care systems. The cardiometabolic clinic would facilitate interdisciplinary collaboration between different professionals to enable a comprehensive treatment of the cardiometabolic patient.

Cardiometabolic medicine as a distinct medical subspecialty has to be approved by the American Board of Internal Medicine. Until then, cardiometabolic medicine modules could be introduced in medical school, as well as offering continuous medical education (CME) and other cardiometabolic medicine certification programs to physicians. Thereafter, the cardiometabolic physician training program 
has to fully develop and successfully educate cardiometabolic specialists. To create a cardiometabolic clinic, a more detailed analysis of financial models and management strategies in this setting is necessary. Further research on the benefits for patient experience and outcomes of preventive care clinics is required. Once the cardiometabolic clinic is established, we hope to expand this model by encouraging research in the field of cardiometabolic medicine.

\section{Compliance with Ethical Standards}

Conflict of Interest The authors declare that they have no conflicts of interest relevant to the content of this manuscript.

Human and Animal Rights and Informed Consent This article does not contain any studies with human or animal subjects performed by any of the authors.

\section{References}

\section{Papers of particular interest, published recently, have been highlighted as: • Of importance •• Of major importance}

1. CDC. National Diabetes Statistics Report 2020. Estimates of diabetes and its burden in the United States 2020.

2. Fryar CD, Chen TC, Li X. Prevalence of uncontrolled risk factors for cardiovascular disease: United States, 1999-2010. NCHS Data Brief. 2012;2012:1-8.

3. Ferrannini G, De Bacquer D, De Backer G, Kotseva K, Mellbin L, Wood D, et al. Screening for glucose perturbations and risk factor management in dysglycemic patients with coronary artery diseaseda persistent challenge in need of substantial improvement: a report from ESC EORP Euroaspire V. Diabetes Care. 2020;43(4): 726-33.

4. Lowe LP, Liu K, Greenland P, Metzger BE, Dyer AR, Stamler J. Diabetes, asymptomatic hyperglycemia, and 22-year mortality in black and white men: the Chicago Heart Association Detection Project in Industry study. Diabetes Care. 1997;20(2):163-9.

5. Sidney S, Quesenberry CP, Jaffe MG, Sorel M, Nguyen-Huynh MN, Kushi LH, et al. Recent trends in cardiovascular mortality in the United States and public health goals. JAMA Cardiol. 2016 Aug 1;1(5):594-9.

6. McClellan M, Brown N, Califf RM, Warner JJ. Call to action: urgent challenges in cardiovascular disease: a presidential advisory from the American Heart Association. Circulation. 2019 Feb 26 [cited 2020 Aug 10];139(9):E44-54. Available from: https:// www.ahajournals.org/doi/10.1161/CIR.0000000000000652

7. 2020 Heart Disease and Stroke Statistical Update Fact Sheet At-aGlance Heart Disease, Stroke and other cardiovascular diseases. available from: https://www.heart.org/-/media/files/about-us/ statistics/2020-heart-disease-and-stroke-ucm_505473.pdf?la=en. accessed 8.30.2020.

8. Hales CM, Fryar CD, Carroll MD, Freedman DS, Ogden CL. Trendsinobesity and severe obesity prevalence in usyouth and adultsby sex and age, 2007-2008 to 2015-2016. JAMA - J Am Med Assoc. 2018 Apr 24 [cited 2020 Aug 11];319(16):1723-5. Available from: https://figshare.com/s/276f9dc0b37d8ecd0ab0.
9. Ward ZJ, Bleich SN, Cradock AL, Barrett JL, Giles CM, Flax C, et al. Projected U.S. state-level prevalence of adult obesity and severe obesity. N Engl J Med. 2019;381(25):2440-50.

10. Abdullah A, Wolfe R, Stoelwinder JU, de Courten M, Stevenson C, Walls HL, et al. The number of years lived with obesity and the risk of all-cause and cause-specific mortality. Int J Epidemiol. 2011;40(4):985-96.

11. Benjamin EJ, Muntner P, Alonso A, Bittencourt MS, Callaway CW, Carson AP, et al. Heart disease and stroke statistics-2019 update: a report from the American Heart Association. Vol. 139, Circulation. 2019. 56-528 p.

12. Guo F, Garvey, Timothy W. Trends in cardiovascular health metrics in obese adults: National Health and Nutrition Examination Survey (NHANES). [cited 2020 Mar 19];1988-2014. Available from: http://jaha.ahajournals.org/content/5/7/e003619/DC1/ embed/inline-supplementarymaterial-1.pdf

13. Sarwar N, Gao P, Kondapally Seshasai SR, Gobin R, Kaptoge S, Di Angelantonio E, et al. Diabetes mellitus, fasting blood glucose concentration, and risk of vascular disease: a collaborative metaanalysis of 102 prospective studies. Lancet. 2010 Jun 26 [cited 2020 Aug 11];375(9733):2215-22. Available from: www. thelancet.com

14. Benjamin EJ, Blaha MJ, Chiuve SE, Cushman M, Das SR, Deo R, et al. Heart disease and stroke statistics - 2017 update: a report from the American Heart Association. Circulation. 2017 Mar 7 [cited 2019 Sep 4];135(10). Available from: https://www.ahajournals. org/doi/10.1161/CIR.0000000000000485

15. Suskin N, McKelvie RS, Burns RJ, Latini R, Pericak D, Probstfield $\mathrm{J}$, et al. Glucose and insulin abnormalities relate to functional capacity in patients with congestive heart failure. Eur Heart J. 2000;21(16):1368-75.

16. Fox CS, Larson MG, Leip EP, Culleton B, Wilson PWF, Levy D. Predictors of new-onset kidney disease in a community-based population. J Am Med Assoc. 2004;291(7):844-50.

17. Fox CS, Matsushita K, Woodward M, Bilo HJG, Chalmers J, Lambers Heerspink HJ, et al. Associations of kidney disease measures with mortality and end-stage renal disease in individuals with and without diabetes: a meta-analysis. Lancet. 2012;381(9864): 374.

18. Chu M, Wang AYM, Chan IHS, Chui SH, Lam CWK. Serum small-dense LDL abnormalities in chronic renal disease patients. Br J Biomed Sci. 2012;69(3):99-102.

19. de Boer IH, Astor BC, Kramer H, Palmas W, Rudser K, Seliger SL, et al. Mild elevations of urine albumin excretion are associated with atherogenic lipoprotein abnormalities in the Multi-Ethnic Study of Atheroslcerosis (MESA). Atherosclerosis. 2008;197(1):407-14.

20. Marso SP, Daniels GH, Brown-Frandsen K, Kristensen P, Mann JFE, Nauck MA, et al. Liraglutide and cardiovascular outcomes in type 2 diabetes. N Engl J Med. 2016 Jul 28 [cited 2019 Jul 2];375(4):311-22. Available from: http://www.ncbi.nlm.nih. gov/pubmed/27295427.

21.• Neal B, Perkovic V, Mahaffey KW, de Zeeuw D, Fulcher G, Erondu N, et al. Canagliflozin and cardiovascular and renal events in type 2 diabetes. N Engl J Med. 2017 [cited 2019 Jul 2];377(7): 644-57. Available from: http://www.ncbi.nlm.nih.gov/pubmed/ 28605608. Findings from this study demonstrate a significant reduction of primary composite outcome of cardiovascularrelated death, nonfatal myocardial infarction, and nonfatal stroke. Additionally, renal outcomes of diabetic patients were improved as well. This trial showed the benefits of canagfliflozin for renal as well as cardiovascular outcomes.

22. FDA approves Ozempic ${ }^{\circledR}$ for cardiovascular risk reduction in adults with type 2 diabetes and known heart disease, updates Rybelsus ${ }^{\circledR}$ label. [cited $2020 \mathrm{Jul}$ 15]. Available from: https:// www.novonordisk-us.com/media/news-releases.html?122981 
23. Gerstein HC, Colhoun HM, Dagenais GR, Diaz R, Lakshmanan M, Pais P, et al. Dulaglutide and cardiovascular outcomes in type 2 diabetes (REWIND): a double-blind, randomised placebocontrolled trial. Lancet (London, England). 2019 [cited 2019 Jul 20];394(10193):121-30. Available from: http://www.ncbi. nlm.nih.gov/pubmed/31189511. This study investigated the effects of dulaglutide when added to existing antihyperglycemic regimens of individuals with type 2 diabetes on cardiovascular outcomes. While all-cause mortality did not differ between the groups, the incidence of primary cardiovascular outcome was significantly lower in the dulaglutide group than the placebo group.

24. Zinman B, Wanner C, Lachin JM, Fitchett D, Bluhmki E, Hantel S, et al. Empagliflozin, cardiovascular outcomes, and mortality in type 2 diabetes. N Engl J Med. 2015 Nov 26 [cited 2019 Jul 2];373(22): 2117-28. Available from: http://www.ncbi.nlm.nih.gov/pubmed/ 26378978.

25. FDA Drug Safety Communication. FDA approves new treatment for a type of heart failure | FDA. 2020 [cited 2020 Jul 15]. Available from: https://www.fda.gov/news-events/press-announcements/fdaapproves-new-treatment-type-heart-failure

26. McMurray JJV, Solomon SD, Inzucchi SE, Kober L, Kosiborod MN, Martinez FA, et al. Dapagliflozin in patients with heart failure and reduced ejection fraction. N Engl J Med. 2019;381(21):19952008.

27. Shyangdan DS, Royle P, Clar C, Sharma P, Waugh N, Snaith A. Glucagon-like peptide analogues for type 2 diabetes mellitus. Cochrane database Syst Rev. 2011 Oct 5 [cited 2019 Jun 26];(10):CD006423. Available from: http://doi.wiley.com/10. 1002/14651858.CD006423.pub2

28. Arnold S V, de Lemos JA, Rosenson RS, Ballantyne CM, Liu Y, Mues KE, et al. Use of guideline-recommended risk reduction strategies among patients with diabetes and atherosclerotic cardiovascular disease. Circulation. 2019 Aug 13 [cited 2020 mar 31];140(7): 618-20. Available from: http://www.ncbi.nlm.nih.gov/pubmed/ 31174429 .

29. Saxon DR, Reiter-Brennan C, Blaha MJ, Eckel RH. Cardiometabolic medicine: development of a new subspecialty. J Clin Endocrinol Metab. 2020;105(7):dgaa261 In this publication, we outline the importance of establishing a field of cardiometabolic medicine and develop a proposal for a cardiometabolic training program for doctors looking to specialize in this field.

30. Eden J, Berwick D, Wilensky G. Graduate medical education that meets the nation's health needs. Graduate Medical Education That Meets the Nation's Health Needs 2014

31. Fan W, Song Y, Inzucchi SE, Sperling L, Cannon CP, Arnold S V., et al. Composite cardiovascular risk factor target achievement and its predictors in US adults with diabetes: the Diabetes Collaborative Registry. Diabetes, Obes Metab. 2019 May 14 [cited 2020 Aug 16];21(5):1121-7. Available from: https://onlinelibrary. wiley.com/doi/abs/10.1111/dom.13625

32. Miele L, Targher G. Understanding the association between developing a fatty liver and subsequent cardio-metabolic complications. Vol. 9, Expert Review of Gastroenterology and Hepatology. Taylor and Francis Ltd; 2015 [cited 2020 Aug 30]. p. 1243-5. Available from: https://pubmed.ncbi.nlm.nih.gov/26224399/

33. Andreatta P, Marzano D. Healthcare management strategies. Curr Opin Obstet Gynecol. 2012 [cited 2020 Aug 12];24(6):445-52. Available from: http://journals.lww.com/00001703-20121200000015

34. Fradgley EA, Paul CL, Bryant J. A systematic review of barriers to optimal outpatient specialist services for individuals with prevalent chronic diseases: what are the unique and common barriers experienced by patients in high income countries? Int J Equity Health. 2015 [cited 2020 Aug 12];14(1):52. Available from: https:// equityhealthj.biomedcentral.com/articles/10.1186/s12939-0150179-6

35. Schoen C, Osborn R, How SKH, Doty MM, Peugh J. In chronic condition: experiences of patients with complex health care needs, in eight countries, 2008. Health Aff. 2009;28(1).

36. Potthoff SA, Vonend O. Multidisciplinary approach in the treatment of resistant hypertension. Curr Hypertens Rep. 2017;19(1):9.

37. Virani SS, Akeroyd JM, Ramsey DJ, Chan WJ, Frazier L, Nasir K, et al. Comparative effectiveness of outpatient cardiovascular disease and diabetes care delivery between advanced practice providers and physician providers in primary care: implications for care under the Affordable Care Act. Am Heart J. 2016;181:74-82.

38. Mokdad AH, Marks JS, Stroup DF, Gerberding JL. Actual Causes of Death in the United States, 2000. J Am Med Assoc. American Medical Association. 2004;291:1238-45.

39. Lobelo F, Duperly J, Frank E. Physical activity habits of doctors and medical students influence their counselling practices. Br J Sports Med. 2009;43(2):89-92.

40. Eckel RH. Preventive cardiology by lifestyle intervention: Opportunity and/or challenge? Presidential address at the 2005 American Heart Association scientific sessions. In: Circulation. Circulation; 2006 [cited 2020 Aug 31]. p. 2657-61. Available from: https://pubmed.ncbi.nlm.nih.gov/16754814/

41. Merz CNB, Alberts MJ, Balady GJ, Ballantyne CM, Berra K, Black HR, et al. ACCF/AHA/ACP 2009 competence and training statement: a curriculum on prevention of cardiovascular disease: a report of the American College of Cardiology Foundation/American Heart Association/American College of Physicians Task Force on Competence And Training (writing committee to develop a competence and training statement on prevention of cardiovascular disease). Vol. 120, Circulation. 2009.

42. Fisher L, Gonzalez JS, Polonsky WH. The confusing tale of depression and distress in patients with diabetes: a call for greater clarity and precision. Diabet Med. 2014;31(7):764-72.

43. Naderi SH, Bestwick JP, Wald DS. Adherence to drugs that prevent cardiovascular disease: meta-analysis on 376,162 patients. Am J Med. 2012;125(9):882-887.e1.

44. McEwen A, West R, McRobbie H. Effectiveness of specialist group treatment for smoking cessation vs. one-to-one treatment in primary care. Addict Behav. 2006 [cited 2020 Aug 13];31(9): 1650-60. Available from: https://pubmed.ncbi.nlm.nih.gov/ 16443331/

45. Stead LF, Perera R, Bullen C, Mant D, Hartmann-Boyce J, Cahill $\mathrm{K}$, et al. Nicotine replacement therapy for smoking cessation. Cochrane Database Syst Rev. 2012;11:CD000146.

46. Odphp. 2015-2020 Dietary guidelines for Americans. 2015 [cited 2020 Aug 13]. Available from: http://health.gov/dietaryguidelines/ 2015/guidelines/.

47. Estruch R, Ros E, Salas-Salvadó J, Covas MI, Corella D, Arós F, et al. Primary prevention of cardiovascular disease with a Mediterranean diet. N Engl J Med. 2013;378(25):e34.

48. Eckel RH, Jakicic JM, Ard JD, De Jesus JM, Houston Miller N, Hubbard VS, et al. 2013 AHA/ACC guideline on lifestyle management to reduce cardiovascular risk: a report of the American college of cardiology/American heart association task force on practice guidelines. J Am Coll Cardiol. 2014;63(25 PART B):2960-84.

49. American Diabetes Association. Standards of medical care in diabetes-2020. Care Diabetes J. 2020.

50. Franz MJ, MacLeod J, Evert A, Brown C, Gradwell E, Handu D, et al. Academy of nutrition and dietetics nutrition practice guideline for type 1 and type 2 diabetes in adults: systematic review of evidence for medical nutrition therapy effectiveness and recommendations for integration into the nutrition care process. J Acad Nutr Diet. 2017;117(10):1659-79.

51. Norris SL, Lau J, Smith SJ, Schmid CH, Engelgau MM. Selfmanagement education for adults with type 2 diabetes. A meta- 
analysis of the effect on glycemic control. Diabetes Care. 2002;25(7):1159-71.

52. He X, Li J, Wang B, Yao Q, Li L, Song R, et al. Diabetes selfmanagement education reduces risk of all-cause mortality in type 2 diabetes patients: a systematic review and meta-analysis. Endocrine. 2017;55(3):712-31.

53. Cooke D, Bond R, Lawton J, Rankin D, Heller S, Clark M, et al. Structured type 1 diabetes education delivered within routine care: impact on glycemic control and diabetes-specific quality of life. Diabetes Care. 2013;36(2):270-2.

54. Strawbridge LM, Lloyd JT, Meadow A, Riley GF, Howell BL. Use of Medicare's diabetes self-management training benefit. Health Educ Behav. 2015;42(4):530-8.

55. Li R, Shrestha SS, Lipman R, Burrows NR, Kolb LE, Rutledge S, et al. Diabetes self-management education and training among privately insured persons with newly diagnosed diabetes-United States, 2011-2012. MMWR Morb Mortal Wkly Rep. 2014;63(46):1045-9.

56. Suaya JA, Shepard DS, Normand SLT, Ades PA, Prottas J, Stason WB. Use of cardiac rehabilitation by Medicare beneficiaries after myocardial infarction or coronary bypass surgery. Circulation. 2007;116(15):1653-62.

57. Polgreen LA, Han J, Carter BL, Ardery GP, Coffey CS, Chrischilles EA, et al. Cost-effectiveness of a physicianpharmacist collaboration intervention to improve blood pressure control. Hypertension. 2015 [cited 2020 Aug 31];66(6):1145-51. Available from: https://www.ahajournals.org/doi/10.1161/ HYPERTENSIONAHA.115.06023

58. Carter BL, Rogers M, Daly J, Zheng S, James PA. The potency of team-based care interventions for hypertension: a meta-analysis. Arch Intern Med. American Medical Association; 2009 [cited 2020 Aug 31];169:1748-55. Available from: https://jamanetwork. $\mathrm{com} /$

59. Ho PM, Bryson CL, Rumsfeld JS. Medication adherence: its importance in cardiovascular outcomes. Circulation. 2009;119(23): 3028-35.

60. Warden BA, Shapiro MD, Fazio S. The role of the clinical pharmacist in a preventive cardiology practice. Ann Pharmacother. 2019 [cited 2020 Aug 31];53(12):1214-9. Available from: http:// journals.sagepub.com/doi/10.1177/1060028019864669

61. De Ferranti SD, De Boer IH, Fonseca V, Fox CS, Golden SH, Lavie $\mathrm{CJ}$, et al. Type 1 diabetes mellitus and cardiovascular disease: a scientific statement from the American Heart Association and American Diabetes Association. Diabetes Care. 2014 [cited 2020 Aug 12];37(10):2843-63. Available from: http://care. diabetesjournals.org/lookup/

62. Sperling LS, Mechanick JI, Neeland IJ, Herrick CJ, Després JP, Ndumele CE, et al. The CardioMetabolic Health Alliance working toward a new care model for the metabolic syndrome. J Am Coll Cardiol. 2015;66(9):1050-67.

63. Dabelea D, Stafford JM, Mayer-Davis EJ, D’Agostino R, Dolan L, Imperatore $\mathrm{G}$, et al. Association of type 1 diabetes vs type 2 diabetes diagnosed during childhood and adolescence with complications during teenage years and young adulthood. JAMA J Am Med Assoc. 2017;317(8):825-35.

64. Change Healthcare. Finding the value: the state of value-based care in 2018. 2018.

65. Jiang HJ, Russo CA, Barrett ML. Nationwide frequency and costs of potentially preventable hospitalizations, 2006: Statistical Brief \#72. Healthcare Cost and Utilization Project (HCUP) Statistical Briefs. 2006 [Cited 2020 Aug 20]. Available from: http://www. ncbi.nlm.nih.gov/pubmed/21510033.

66. Hutchison J, Thompson ME, Troyer J, Elnitsky C, Coffman MJ, Lori TM. The effect of North Carolina free clinics on hospitalizations for ambulatory care sensitive conditions among the uninsured. BMC Health Serv Res. 2018;18(1):280.

67. Beckman AL, Becerra AZ, Marcus A, Annette DuBard C, Lynch $\mathrm{K}$, Maxson E, et al. Medicare annual wellness visit association with healthcare quality and costs. Am J Manag Care. 2019;25(3):e7682 .

Publisher's Note Springer Nature remains neutral with regard to jurisdictional claims in published maps and institutional affiliations. 